\title{
Numerical Algorithms for Computing Eigenvalues of Discontinuous Dirac System Using Sinc-Gaussian Method
}

\author{
A. H. Bhrawy, ${ }^{1,2}$ M. M. Tharwat, ${ }^{1,2}$ and A. Al-Fhaid ${ }^{1}$ \\ ${ }^{1}$ Department of Mathematics, Faculty of Science, King Abdulaziz University, Jeddah 21589, Saudi Arabia \\ ${ }^{2}$ Department of Mathematics, Faculty of Science, Beni-Suef University, Beni-Suef 62511, Egypt
}

Correspondence should be addressed to A. H. Bhrawy, alibhrawy@yahoo.co.uk

Received 8 March 2012; Accepted 26 May 2012

Academic Editor: Zhenya Yan

Copyright (C) 2012 A. H. Bhrawy et al. This is an open access article distributed under the Creative Commons Attribution License, which permits unrestricted use, distribution, and reproduction in any medium, provided the original work is properly cited.

The eigenvalues of a discontinuous regular Dirac systems with transmission conditions at the point of discontinuity are computed using the sinc-Gaussian method. The error analysis of this method for solving discontinuous regular Dirac system is discussed. It shows that the error decays exponentially in terms of the number of involved samples. Therefore, the accuracy of the new method is higher than the classical sinc-method. Numerical results indicating the high accuracy and effectiveness of these algorithms are presented. Comparisons with the classical sinc-method are given.

\section{Introduction}

The mathematical modeling of many practical problems in mechanics and other areas of mathematical physics requires solutions of boundary value problems (see, e.g., [1-7]). Boundary value problems with discontinuity conditions arise in different branches of mathematics, mechanics, radio, electronics, geophysics, and other fields of natural science and technology. For example, discontinuous conditions inside an interval are connected with discontinuous or nonsmooth properties of media (see [8-11] and references there in).

Sampling theory is one of the most important mathematical tools used in communication engineering since it enables engineers to reconstruct signals from some of their sampled data. A fundamental result in information theory is the Whittaker-Kotel'nikovShannon (WKS) sampling theorem [12-14]. It states that any $f \in P W_{\sigma}^{2}$, where $P W_{\sigma}^{2}$ is the space of all entire functions of exponential type $\sigma>0$ which lie in $L^{2}(\mathbb{R})$ when restricted to $\mathbb{R}$, can be reconstructed from its sampled values $\{f(n \pi / \sigma): n \in \mathbb{Z}\}$ by the formula

$$
f(\lambda)=\sum_{n \in \mathbb{Z}} f\left(\frac{n \pi}{\sigma}\right) \operatorname{sinc}(\sigma \lambda-n \pi), \quad \lambda \in \mathbb{C},
$$


where

$$
\text { sinc }(\sigma \curlywedge-n \pi):= \begin{cases}\frac{\sin (\sigma \lambda-n \pi)}{(\sigma \lambda-n \pi)}, & \lambda \neq \frac{n \pi}{\sigma}, \\ 1, & \lambda=\frac{n \pi}{\sigma} .\end{cases}
$$

Series (1.1) converges absolutely and uniformly on compact subsets of $\mathbb{C}$, and uniform on $\mathbb{R}$, cf. [15]. Expansion (1.1) is used in several approximation problems which are known as sinc methods, see, for example, [16-19]. In particular the sinc-method is used to approximate eigenvalues of boundary value problems, see, for examples, [20-24]. The sinc-method has a slow rate of decay at infinity, which is as slow as $O\left(\left|\lambda^{-1}\right|\right)$. There are several attempts to improve the rate of decay. One of the interesting ways is to multiply the sinc-function in (1.1) by a kernel function, see, for example, [25-27]. Let $h \in(0, \pi / \sigma]$ and $\gamma \in(0, \pi-h \sigma)$. Assume that $\Phi \in P W_{\gamma}^{2}$ such that $\Phi(0)=1$, then for $f \in P W_{\sigma}^{2}$ we have the expansion [28],

$$
f(\lambda)=\sum_{n=-\infty}^{\infty} f(n h) \operatorname{sinc}\left(h^{-1} \pi \lambda-n \pi\right) \Phi\left(h^{-1} \lambda-n\right) .
$$

The speed of convergence of the series in (1.3) is determined by the decay of $|\Phi(\lambda)|$. But the decay of an entire function of exponential type cannot be as fast as $e^{-c|x|}$ as $|x| \rightarrow \infty$, for some positive $c$, [28]. In [29], Qian has introduced the following regularized sampling formula. For $h \in(0, \pi / \sigma], N \in \mathbb{N}$ and $r>0$,

$$
\left(G_{h, N} f\right)(x)=\sum_{n \in Z_{N}(x)} f(n h) S_{n}\left(h^{-1} \pi x\right) G\left(\frac{x-n h}{\sqrt{2} r h}\right), \quad x \in \mathbb{R}
$$

where $G(t):=\exp \left(-t^{2}\right)$, which is called the Gaussian function, $S_{n}\left(h^{-1} \pi x\right):=\operatorname{sinc}\left(h^{-1} \pi x-\right.$ $n \pi), Z_{N}(x):=\left\{n \in \mathbb{Z}:\left|\left[h^{-1} x\right]-n\right| \leq N\right\}$ and $[x]$ denotes the integer part of $x \in \mathbb{R}$, see also [30, 31]. Qian also derived the following error bound. If $f \in P W_{\sigma}^{2}, h \in(0, \pi / \sigma]$ and $a:=\min \{r(\pi-h \sigma),(N-2) / r\} \geq 1$, then

$$
\left|f(x)-\left(G_{h, N} f\right)(x)\right| \leq \frac{2 \sqrt{\sigma \pi}\|f\|_{2}}{\pi^{2} a^{2}}\left(\sqrt{2 \pi} a+e^{3 / 2 r^{2}}\right) e^{-a^{2} / 2}, \quad x \in \mathbb{R} .
$$

In [28], Schmeisser and Stenger extended the operator (1.4) to the complex domain $\mathbb{C}$. For $\sigma>0, h \in(0, \pi / \sigma]$ and $\omega:=(\pi-h \sigma) / 2$, they defined the operator [28],

$$
\left(\mathcal{G}_{h, N} f\right)(\lambda):=\sum_{n \in \mathbb{Z}_{N}(\lambda)} f(n h) S_{n}\left(\frac{\pi \lambda}{h}\right) G\left(\frac{\sqrt{\omega}(\lambda-n h)}{\sqrt{N} h}\right),
$$

where $\mathbb{Z}_{N}(\lambda):=\left\{n \in \mathbb{Z}:\left|\left[h^{-1} \mathfrak{R} \lambda+1 / 2\right]-n\right| \leq N\right\}$ and $N \in \mathbb{N}$. Note that the summation limits in (1.6) depend on the real part of $\lambda$. Schmeisser and Stenger, [28], proved that if $f$ is an entire 
function of exponential type $\sigma>0$, then for $h \in(0, \pi / \sigma), \omega:=(\pi-h \sigma) / 2, N \in \mathbb{N},|\Im \lambda|<N$, we have

$$
\left|f(\lambda)-\left(\mathcal{G}_{h, N} f\right)(\lambda)\right| \leq 2\left|\sin \left(h^{-1} \pi \lambda\right)\right|\|f\|_{\infty} \frac{e^{-\omega N}}{\sqrt{\pi \omega N}} \beta_{N}\left(h^{-1} \Im \curlywedge\right), \quad \lambda \in \mathbb{C}
$$

where

$$
\beta_{N}(t):=\cosh (2 \omega t)+\frac{2 e^{\omega t^{2} / N}}{\sqrt{\pi \omega N}\left[1-(t / N)^{2}\right]}+\frac{1}{2}\left[\frac{e^{2 \omega t}}{e^{2 \pi(N-t)}-1}+\frac{e^{-2 \omega t}}{e^{2 \pi(N+t)}-1}\right]
$$

The amplitude error arises when the exact values $f(n h)$ of (1.6) are replaced by the approximations $\tilde{f}(n h)$. We assume that $\tilde{f}(n h)$ are close to $f(n h)$, that is, there is $\varepsilon>0$, sufficiently small such that

$$
\sup _{n \in \mathbb{Z}_{n}(\lambda)}|f(n h)-\tilde{f}(n h)|<\varepsilon
$$

Let $h \in(0, \pi / \sigma), \omega:=(\pi-h \sigma) / 2$ and $N \in \mathbb{N}$ be fixed numbers. The authors in [9] proved that if (1.9) is held, then for $|\Im \lambda|<N$, we have

$$
\left|\left(\mathcal{G}_{h, N} f\right)(\lambda)-\left(\mathcal{G}_{h, N} \tilde{f}\right)(\lambda)\right| \leq A_{\varepsilon, N}(\Im \lambda)
$$

where

$$
A_{\varepsilon, N}(\Im \lambda)=2 \varepsilon e^{-\omega / 4 N}\left(1+\sqrt{\frac{N}{\omega \pi}}\right) \exp \left((\omega+\pi) h^{-1}|\Im \lambda|\right)
$$

We are concerned with the computation of eigenvalues the Dirac system

$$
\begin{gathered}
u_{2}^{\prime}(x, \lambda)-p_{1}(x) u_{1}(x, \lambda)=\lambda u_{1}(x, \lambda), \\
u_{1}^{\prime}(x, \lambda)+p_{2}(x) u_{2}(x, \lambda)=-\lambda u_{2}(x, \lambda), \\
x \in[-1,0) \cup(0,1]
\end{gathered}
$$

with boundary conditions

$$
\begin{gathered}
U_{1}(\mathbf{u}):=\sin \alpha u_{1}(-1, \lambda)+\cos \alpha u_{2}(-1, \lambda)=0, \\
U_{2}(\mathbf{u}):=\sin \beta u_{1}(1, \lambda)+\cos \beta u_{2}(1, \lambda)=0,
\end{gathered}
$$


and transmission conditions

$$
\begin{aligned}
& U_{3}(\mathbf{u}):=u_{1}\left(0^{-}, \lambda\right)-\delta u_{1}\left(0^{+}, \lambda\right)=0, \\
& U_{4}(\mathbf{u}):=u_{2}\left(0^{-}, \lambda\right)-\delta u_{2}\left(0^{+}, \lambda\right)=0,
\end{aligned}
$$

where $\lambda \in \mathbb{C}$; the real valued function $p_{1}(\cdot)$ and $p_{2}(\cdot)$ are continuous in $[-1,0)$ and $(0,1]$, and have finite limits $p_{1}\left(0^{ \pm}\right):=\lim _{x \rightarrow 0^{ \pm}} p_{1}(x), p_{2}\left(0^{ \pm}\right):=\lim _{x \rightarrow 0^{ \pm}} p_{2}(x) ; \delta \in \mathbb{R}, \alpha, \beta \in[0, \pi)$ and $\delta \neq 0$.

In this paper we will use the sinc-Gaussian sampling formula (1.6) to compute eigenvalues of the Dirac system (1.12)-(1.16). As expected, the new method reduced the error bounds remarkably, see examples at the end of this paper. Special attention is given to the comparison of the numerical results obtained by the new method with those found by classical sinc-method. We would like to mention that works in direction of computing eigenvalues with the sinc-Gaussian, are few, see for example, [9]. Also papers in computing of eigenvalues with discontinuous are few, see [10,32]. However, the computing of eigenvalues by sinc-Gaussian technique which has discontinuity conditions, do not exist as for as we know.

The paper is organized as follows: Section 2 contains some preliminary results and the approximated values of the eigenvalues of the Dirac system with discontinuous. The method with error estimates are contained in Section 3. The last section involves some illustrative examples for showing the high accuracy of the proposed technique.

\section{The Approximated Eigenvalues of Dirac System}

In this section we derive approximate values of the eigenvalues of problem (1.12)-(1.16). Recall that problem (1.12)-(1.16) has denumerable set of real and simple eigenvalues, compare with $[33,34]$. Let

$$
\phi(\cdot, \lambda)=\left(\begin{array}{l}
\phi_{1}(\cdot, \lambda) \\
\phi_{2}(\cdot, \lambda)
\end{array}\right), \quad \phi_{i}(x, \lambda)=\left\{\begin{array}{ll}
\phi_{i 1}(x, \lambda), & x \in[-1,0), \\
\phi_{i 2}(x, \lambda), & x \in(0,1],
\end{array} \quad i=1,2\right.
$$

be the solution of (1.12) satisfying the following initial conditions:

$$
\left(\begin{array}{ll}
\phi_{11}(-1, \lambda) & \phi_{12}\left(0^{+}, \lambda\right) \\
\phi_{21}(-1, \lambda) & \phi_{22}\left(0^{+}, \lambda\right)
\end{array}\right)=\left(\begin{array}{cc}
\cos \alpha & \delta^{-1} \phi_{11}\left(0^{-}, \lambda\right) \\
-\sin \alpha & \delta^{-1} \phi_{21}\left(0^{-}, \lambda\right)
\end{array}\right)
$$

Since $\phi(\cdot, \lambda)$ satisfies $(1.13)$, then the eigenvalues of the problem (1.12)-(1.16) are the zeros of the function

$$
\Delta(\lambda)=-\delta^{2}\left(\sin \beta \phi_{12}(1, \lambda)+\cos \beta \phi_{22}(1, \lambda)\right)
$$


Notice that both $\phi(\cdot, \lambda)$ and $\Delta(\lambda)$ are entire functions of $\lambda$ and $\phi(\cdot, \lambda)$ satisfies the system of integral equations, see [32, 35],

$$
\begin{aligned}
& \phi_{11}(x, \lambda)=\cos (\lambda(x+1)-\alpha)-\tau_{-1,1} \phi_{11}(x, \lambda)-\tilde{\tau}_{-1,2} \phi_{21}(x, \lambda), \\
& \phi_{21}(x, \lambda)=\sin (\lambda(x+1)-\alpha)+\tilde{\tau}_{-1,1} \phi_{11}(x, \lambda)-\tau_{-1,2} \phi_{21}(x, \lambda), \\
& \phi_{12}(x, \lambda)= \frac{1}{\delta} \phi_{11}\left(0^{-}, \lambda\right) \cos (\lambda x)-\frac{1}{\delta} \phi_{21}\left(0^{-}, \lambda\right) \sin (\lambda x) \\
&-\tau_{0,1} \phi_{12}(x, \lambda)-\tilde{\tau}_{0,2} \phi_{22}(x, \lambda), \\
& \phi_{22}(x, \lambda)= \frac{1}{\delta} \phi_{11}\left(0^{-}, \lambda\right) \sin (\lambda x)+\frac{1}{\delta} \phi_{21}\left(0^{-}, \lambda\right) \cos (\lambda x) \\
&+\tilde{\tau}_{0,1} \phi_{12}(x, \lambda)-\tau_{0,2} \phi_{22}(x, \lambda),
\end{aligned}
$$

where $\tau_{-1, i}, \tilde{\tau}_{-1, i}, \tau_{0, i}$ and $\tilde{\tau}_{0, i}, i=1,2$, are the Volterra integral operators defined by

$$
\begin{aligned}
& \tau_{-1,1} \varphi(x, \lambda):=\int_{-1}^{x} \sin \lambda(x-t) p_{1}(t) \varphi(t, \lambda) d t, \\
& \tau_{-1,2} \varphi(x, \lambda):=\int_{-1}^{x} \sin \lambda(x-t) p_{2}(t) \varphi(t, \lambda) d t, \\
& \tilde{\tau}_{-1,1} \varphi(x, \lambda):=\int_{-1}^{x} \cos \lambda(x-t) p_{1}(t) \varphi(t, \lambda) d t, \\
& \tilde{\tau}_{-1,2} \varphi(x, \lambda):=\int_{-1}^{x} \cos \lambda(x-t) p_{2}(t) \varphi(t, \lambda) d t, \\
& \tau_{0,1} \varphi(x, \lambda):=\int_{0}^{x} \sin \lambda(x-t) p_{1}(t) \varphi(t, \lambda) d t, \\
& \tau_{0,2} \varphi(x, \lambda):=\int_{0}^{x} \sin \lambda(x-t) p_{2}(t) \varphi(t, \lambda) d t, \\
& \tilde{\tau}_{0,1} \varphi(x, \lambda):=\int_{0}^{x} \cos \lambda(x-t) p_{1}(t) \varphi(t, \lambda) d t, \\
& \tilde{\tau}_{0,2} \varphi(x, \lambda):=\int_{0}^{x} \cos \lambda(x-t) p_{2}(t) \varphi(t, \lambda) d t .
\end{aligned}
$$

For convenience, we define the constants

$$
\begin{gathered}
c_{1}:=\int_{-1}^{0}\left[\left|p_{1}(t)\right|+\left|p_{2}(t)\right|\right] d t, \quad c_{2}:=c_{1} \exp \left(c_{1}\right), \\
c_{3}:=\int_{0}^{1}\left[\left|p_{1}(t)\right|+\left|p_{2}(t)\right|\right] d t, \quad c_{4}:=c_{2}+\frac{2}{|\delta|}\left(1+c_{2}\right) .
\end{gathered}
$$


Define $f_{-1, i}(\cdot, \lambda)$ and $f_{0, i}(\cdot, \lambda), i=1,2$, to be

$$
\begin{aligned}
f_{-1,1}(x, \lambda) & :=\tau_{-1,1} \phi_{11}(x, \lambda)+\widetilde{\tau}_{-1,2} \phi_{21}(x, \lambda), \\
f_{-1,2}(x, \lambda) & :=\widetilde{\tau}_{-1,1} \phi_{11}(x, \lambda)-\tau_{-1,2} \phi_{21}(x, \lambda), \\
f_{0,1}(x, \lambda) & :=\tau_{0,1} \phi_{12}(x, \lambda)+\widetilde{\tau}_{0,2} \phi_{22}(x, \lambda), \\
f_{0,2}(x, \lambda) & :=\tilde{\tau}_{0,1} \phi_{12}(x, \lambda)-\tau_{0,2} \phi_{22}(x, \lambda) .
\end{aligned}
$$

Lemma 2.1. The functions $f_{-1,1}(x, \lambda)$ and $f_{-1,2}(x, \lambda)$ are entire in $\lambda$ for any fixed $x \in[-1,0)$ and satisfy the growth condition

$$
\left|f_{-1,1}(x, \lambda)\right|, \quad\left|f_{-1,2}(x, \lambda)\right| \leq 2 c_{2} e^{|\Im \lambda|(x+1)}, \quad \lambda \in \mathbb{C} .
$$

Proof. Since $f_{-1,1}(x, \lambda)=\tau_{-1,1} \phi_{11}(x, \lambda)+\tilde{\tau}_{-1,2} \phi_{21}(x, \lambda)$, then from (2.4) and (2.5) we obtain

$$
\begin{aligned}
f_{-1,1}(x, \lambda)= & \tau_{-1,1} \cos (\lambda(x+1)-\alpha)+\tilde{\tau}_{-1,2} \sin (\lambda(x+1)-\alpha)-\tau_{-1,1} f_{-1,1}(x, \lambda) \\
& +\tilde{\tau}_{-1,2} f_{-1,2}(x, \lambda) .
\end{aligned}
$$

Using the inequalities $|\sin z| \leq e^{|\Im z|}$ and $|\cos z| \leq e^{|\Im z|}$ for $z \in \mathbb{C}$, leads for $\lambda \in \mathbb{C}$ to

$$
\begin{aligned}
\left|f_{-1,1}(x, \lambda)\right| \leq & \left|\tau_{-1,1} \cos (\lambda(x+1)-\alpha)\right|+\left|\tilde{\tau}_{-1,2} \sin (\lambda(x+1)-\alpha)\right| \\
& +\left|\tau_{-1,1} f_{-1,1}(x, \lambda)\right|+\left|\tilde{\tau}_{-1,2} f_{-1,2}(x, \lambda)\right| \\
\leq & e^{|\Im \lambda|(x+1)} \int_{-1}^{x}\left[\left|p_{1}(t)\right|\left|f_{-1,1}(t, \lambda)\right|+\left|p_{2}(t)\right|\left|f_{-1,2}(t, \lambda)\right|\right] e^{-|\Im \lambda|(t+1)} d t \\
& +2 e^{|\Im \lambda|(x+1)} \int_{-1}^{x}\left[\left|p_{1}(t)\right|+\left|p_{2}(t)\right|\right] d t \leq 2 c_{1} e^{|\Im \lambda|(x+1)} \\
& +e^{|\Im \lambda|(x+1)} \int_{-1}^{x}\left[\left|p_{1}(t)\right|\left|f_{-1,1}(t, \lambda)\right|+\left|p_{2}(t)\right|\left|f_{-1,2}(t, \lambda)\right|\right] e^{-|\Im \lambda|(t+1)} d t .
\end{aligned}
$$

The above inequality can be reduced to

$$
e^{-|\Im \lambda|(x+1)}\left|f_{-1,1}(x, \lambda)\right| \leq 2 c_{1}+\int_{-1}^{x}\left[\left|p_{1}(t)\right|\left|f_{-1,1}(t, \lambda)\right|+\left|p_{2}(t)\right|\left|f_{-1,2}(t, \lambda)\right|\right] e^{-|\Im \lambda|(t+1)} d t
$$

Similarly, we can prove that

$$
e^{-|\Im \lambda|(x+1)}\left|f_{-1,2}(x, \lambda)\right| \leq 2 c_{1}+\int_{-1}^{x}\left[\left|p_{1}(t)\right|\left|f_{-1,1}(t, \lambda)\right|+\left|p_{2}(t)\right|\left|f_{-1,2}(t, \lambda)\right|\right] e^{-|\Im \lambda|(t+1)} d t
$$

Then from (2.12) and (2.15) and Lemma 3.1 of [34, pp. 204], we obtain (2.12). 
In a similar manner, we will prove the following lemma for $f_{0,1}(\cdot, \lambda)$ and $f_{0,2}(\cdot, \lambda)$.

Lemma 2.2. The functions $f_{0,1}(x, \lambda)$ and $f_{0,2}(x, \lambda)$ are entire in $\lambda$ for any fixed $x \in(0,1]$ and satisfy the growth condition

$$
\left|f_{0,1}(x, \lambda)\right|, \quad\left|f_{0,2}(x, \lambda)\right| \leq 2 c_{3} c_{4} e^{|\Im \lambda|(x+1)}, \quad \lambda \in \mathbb{C} .
$$

Proof. Since $f_{0,1}(x, \lambda)=\tau_{0,1} \phi_{11}(x, \lambda)+\tilde{\tau}_{0,2} \phi_{21}(x, \lambda)$, then from (2.6) and (2.7) we obtain

$$
\begin{aligned}
f_{0,1}(x, \lambda)= & \frac{1}{\delta} \phi_{11}\left(0^{-}, \lambda\right) \tau_{0,1} \cos (\lambda x)-\frac{1}{\delta} \phi_{21}\left(0^{-}, \lambda\right) \tau_{0,1} \sin (\lambda x)-\tau_{0,1} f_{-1,2}(x, \lambda) \\
& +\frac{1}{\delta} \phi_{11}\left(0^{-}, \lambda\right) \tilde{\tau}_{0,2} \sin (\lambda x)+\frac{1}{\delta} \phi_{21}\left(0^{-}, \lambda\right) \tilde{\tau}_{0,2} \cos (\lambda x)+\tilde{\tau}_{0,2} f_{-1,2}(x, \lambda) .
\end{aligned}
$$

Then from (2.4) and (2.5) and Lemma 2.1, we get

$$
\begin{aligned}
f_{0,1}(x, \lambda) \leq & \frac{1}{|\delta|}\left|\phi_{11}\left(0^{-}, \lambda\right)\right|\left|\tau_{0,1} \cos (\lambda x)\right|+\frac{1}{|\delta|}\left|\phi_{21}\left(0^{-}, \lambda\right)\right|\left|\tau_{0,1} \sin (\lambda x)\right|+\left|\tau_{0,1} f_{-1,2}(x, \lambda)\right| \\
& +\frac{1}{|\delta|}\left|\phi_{11}\left(0^{-}, \lambda\right)\right|\left|\tilde{\boldsymbol{\tau}}_{0,2} \sin (\lambda x)\right|+\frac{1}{|\delta|}\left|\phi_{21}\left(0^{-}, \lambda\right)\right|\left|\tilde{\boldsymbol{\tau}}_{0,2} \cos (\lambda x)\right|+\left|\tilde{\tau}_{0,2} f_{-1,2}(x, \lambda)\right| \\
\leq & 2\left(c_{2}+\frac{2}{|\delta|}\left(1+c_{2}\right)\right) c_{3} e^{|\Im \lambda|(x+1)}=2 c_{3} c_{4} e^{|\Im \lambda|(x+1)} .
\end{aligned}
$$

Similarly, we can prove that

$$
f_{0,2}(x, \lambda) \leq 2 c_{3} c_{4} e^{|\Im \lambda|(x+1)} .
$$

\section{The Method and Error Analysis}

In this section we derive the method of computing the eigenvalues of problem (1.12)-(1.16) numerically. We aim to approximate $\Delta(\lambda)$ and hence its zeros, that is, the eigenvalues. The idea is to split $\Delta(\lambda)$ into two parts, one is known and the other is unknown, but is an entire function of exponential type. Then we approximate the unknown part using (1.6) to get the approximate $\Delta(\lambda)$ and then compute the approximate zeros. Now, let us split $\Delta(\lambda)$ into

$$
\Delta(\lambda):=\mathcal{K}(\lambda)+\mathcal{U}(\lambda)
$$

where $\mathcal{U}(\lambda)$ is the unknown part involving integral operators

$$
\begin{aligned}
\mathcal{U}(\lambda):= & \delta^{2} \sin \beta f_{0,1}(1, \lambda)-\delta^{2} \cos \beta f_{0,2}(1, \lambda)-\delta \sin (\lambda-\beta) f_{-1,1}\left(0^{-}, \lambda\right) \\
& -\delta \cos (\lambda+\beta) f_{-1,2}\left(0^{-}, \lambda\right),
\end{aligned}
$$


and $\mathcal{K}(\lambda)$ is the known part

$$
\mathcal{K}(\lambda):=-\delta \sin (2 \lambda-\alpha+\beta)
$$

Then, from Lemmas 2.1 and 2.2, we have the following result.

Lemma 3.1. The function $\mathcal{U}(\lambda)$ is entire in $\lambda$ and the following estimate holds:

$$
|u(\mu)| \leq M e^{2|\Im \lambda|}
$$

where

$$
M:=4|\delta|\left(c_{2}+|\delta| c_{3} c_{4}\right)
$$

Proof. From (3.2), we have

$$
\begin{aligned}
|\mathcal{U}(\lambda)| \leq & |\delta|^{2}|\sin \beta|\left|f_{0,1}(1, \lambda)\right|+|\delta|^{2}|\cos \beta|\left|f_{0,2}(1, \lambda)\right|+|\delta||\sin (\lambda-\beta)|\left|f_{-1,1}\left(0^{-}, \lambda\right)\right| \\
& +|\delta||\cos (\lambda+\beta)|\left|f_{-1,2}\left(0^{-}, \lambda\right)\right| .
\end{aligned}
$$

Using the inequalities $|\sin \lambda| \leq e^{|\Im \lambda|}$ and $|\cos \lambda| \leq e^{|\Im \lambda|}$ for $\lambda \in \mathbb{C}$, and Lemmas 2.1 and 2.2 imply (3.4).

Then $\mathcal{U}(\lambda)$ is an entire function of exponential type 2 . In the following we let $\lambda \in \mathbb{R}$ since all eigenvalues are real. Now we approximate the function $\mathcal{U}(\lambda)$ using the operator (1.6) where $h \in(0, \pi / 2]$ and $\omega:=(\pi-2 h) / 2$ and then we obtain

$$
\left|\mathcal{U}(\lambda)-\left(\mathcal{G}_{h, N} \mathcal{U}\right)(\lambda)\right| \leq T_{h, N}(\lambda)
$$

where

$$
T_{h, N}(\lambda):=2\left|\sin \left(h^{-1} \pi \lambda\right)\right|\|\mathcal{U}\|_{\infty} \frac{e^{-\omega N}}{\sqrt{\pi \omega N}} \beta_{N}(0), \quad \lambda \in \mathbb{R} .
$$

The samples $\mathcal{U}(n h)=\Delta(n h)-\mathcal{K}(n h), n \in \mathbb{Z}_{N}(\lambda)$ cannot be computed explicitly in the general case. We approximate these samples numerically by solving the initial-value problems defined by (1.12) and (2.2) to obtain the approximate values $\tilde{\mathcal{U}}(n h), n \in \mathbb{Z}_{N}(\lambda)$, that is, $\tilde{U}(n h)=\tilde{\Delta}(n h)-\mathcal{K}(n h)$. Accordingly we have the explicit expansion

$$
\left(\mathcal{G}_{h, N} \tilde{u}\right)(\lambda):=\sum_{n \in \mathbb{Z}_{N}(\lambda)} \tilde{u}(n h) S_{n}\left(\frac{\pi \lambda}{h}\right) G\left(\frac{\sqrt{\omega}(\lambda-n h)}{\sqrt{N} h}\right) .
$$

Therefore we get, compare with (1.10),

$$
\left|\left(\mathcal{G}_{h, N} \mathcal{U}\right)(\lambda)-\left(\mathcal{G}_{h, N} \tilde{\mathcal{U}}\right)(\lambda)\right| \leq A_{\varepsilon, N}(0), \quad \lambda \in \mathbb{R}
$$


Now let $\widetilde{\Delta}_{N}(\lambda):=\mathcal{K}(\lambda)+\left(\mathcal{G}_{h, N} \tilde{U}\right)(\lambda)$. From (3.7) and (3.10) we obtain

$$
\left|\Delta(\lambda)-\widetilde{\Delta}_{N}(\lambda)\right| \leq T_{h, N}(\lambda)+A_{\varepsilon, N}(0), \quad \lambda \in \mathbb{R}
$$

Let $\lambda^{*}$ be an eigenvalue and $\lambda_{N}$ be its desired approximation, that is, $\Delta\left(\lambda^{*}\right)=0$ and $\widetilde{\Delta}_{N}\left(\lambda_{N}\right)=$ 0 . From (3.11) we have $\left|\widetilde{\Delta}_{N}\left(\lambda^{*}\right)\right| \leq T_{h, N}\left(\lambda^{*}\right)+A_{\varepsilon, N}(0)$. Now we define an enclosure interval $I_{\varepsilon, N}$ for $\lambda^{*}$. Define the curves

$$
a_{ \pm}(\lambda)=\widetilde{\Delta}_{N}(\lambda) \pm\left(T_{h, N}(\lambda)+A_{\varepsilon, N}(0)\right) .
$$

The curves $a_{+}(\lambda), a_{-}(\lambda)$ trap the curve of $\Delta(\lambda)$ for suitably large $N$. Hence the closure interval is determined by solving $a_{ \pm}(\lambda)=0$, gives an interval $I_{\varepsilon, N}:=\left[a_{-}, a_{+}\right]$. Next we estimate the error $\left|\lambda^{*}-\lambda_{N}\right|$ for the eigenvalue $\lambda^{*}$.

Theorem 3.2. Let $\lambda^{*}$ be an eigenvalue of (1.12)-(1.16) and $\lambda_{N}$ be its approximation. Then, for $\lambda \in \mathbb{R}$, one has the following estimate:

$$
\left|\lambda^{*}-\lambda_{N}\right|<\frac{T_{h, N}\left(\lambda_{N}\right)+A_{\varepsilon, N}(0)}{\inf _{\zeta \in I_{\varepsilon, N}}\left|\Delta^{\prime}(\zeta)\right|}
$$

where the interval $I_{\varepsilon, N}$ is defined above.

Proof. Replacing $\lambda$ by $\lambda_{N}$ in (3.11) we obtain

$$
\left|\Delta\left(\lambda_{N}\right)-\Delta\left(\lambda^{*}\right)\right|<T_{h, N}\left(\lambda_{N}\right)+A_{\varepsilon, N}(0),
$$

where we have used $\widetilde{\Delta}_{N}\left(\lambda_{N}\right)=\Delta\left(\lambda^{*}\right)=0$. Using the mean value theorem yields that for some $\zeta \in J_{\varepsilon, N}:=\left[\min \left(\lambda^{*}, \lambda_{N}\right), \max \left(\lambda^{*}, \lambda_{N}\right)\right]$,

$$
\left|\left(\lambda^{*}-\lambda_{N}\right) \Delta^{\prime}(\zeta)\right| \leq T_{h, N}\left(\lambda_{N}\right)+A_{\varepsilon, N}(0), \zeta \in J_{\varepsilon, N} \subset I_{\varepsilon, N}
$$

Since $\lambda^{*}$ is simple and $N$ is sufficiently large, then $\inf _{\zeta \in I_{\varepsilon, N}}\left|\Delta^{\prime}(\zeta)\right|>0$ and we get (3.13).

\section{Numerical Examples and Comparisons}

This section includes two examples illustrating the sinc-Gaussian method. In the following examples, we consider $\lambda_{k, N}$ being the $k$ th root of $\mathcal{K}(\lambda)+\left(\mathcal{G}_{h, N} \tilde{\mathcal{U}}\right)=0$. Also, it is observed that the approximation $\lambda_{k, N}$ and the exact solution $\lambda_{k}$ are all inside the interval $\left[a_{-}, a_{+}\right]$. We indicate in these two examples the effect of the amplitude error in the proposed method by determining enclosure intervals for different values of $\varepsilon$. All examples are computed in [32] by using the classical sinc method. We see that the sinc-Gaussian method gives remarkably better results. 
Table 1: The absolute errors of sinc-Gaussian and the classical sinc-method with $N=20, h=0.2, \omega=$ 1.3708 .

\begin{tabular}{lccccc}
\hline$\lambda_{k}$ & Sinc & Exact $\lambda_{k}$ & Sinc-Gaussian $\lambda_{k, N}$ & $E$ & $E_{G}$ \\
\hline$\lambda_{-2}$ & -3.5582594249860 & -3.55825932025645 & -3.5582593202564 & $1.04 \times 10^{-7}$ & $2.75 \times 10^{-14}$ \\
$\lambda_{-1}$ & -1.98746291522753 & -1.98746299346156 & -1.98746299346155 & $7.82 \times 10^{-8}$ & $3.77 \times 10^{-15}$ \\
$\lambda_{0}$ & -0.41666654116886 & -0.41666666666666 & -0.41666666666666 & $1.25 \times 10^{-7}$ & $1.83 \times 10^{-15}$ \\
$\lambda_{1}$ & 1.15412963731329 & 1.15412966012822 & 1.15412966012823 & $2.28 \times 10^{-8}$ & $9.77 \times 10^{-15}$ \\
$\lambda_{2}$ & 2.72492581024868 & 2.72492598692312 & 2.72492598692315 & $1.76 \times 10^{-7}$ & $3.28 \times 10^{-14}$ \\
\hline
\end{tabular}

Example 4.1. Consider the system

$$
\begin{gathered}
u_{2}^{\prime}(x)-p(x) u_{1}(x)=\lambda u_{1}(x), \\
u_{1}^{\prime}(x)+p(x) u_{2}(x)=-\lambda u_{2}(x), \quad x \in[-1,0) \cup(0,1],
\end{gathered}
$$

subject to

$$
\begin{array}{cc}
u_{1}(-1)+u_{2}(-1)=0, & u_{1}(1)+u_{2}(1)=0, \\
u_{1}\left(0^{-}\right)-2 u_{1}\left(0^{+}\right)=0, & u_{2}\left(0^{-}\right)-2 u_{2}\left(0^{+}\right)=0 .
\end{array}
$$

Here

$$
p_{1}(x)=p_{2}(x)=p(x)= \begin{cases}x^{2}, & x \in[-1,0) \\ x, & x \in(0,1]\end{cases}
$$

$\alpha=\beta=\pi / 4$ and $\delta=2$.

Direct calculations give

$$
\begin{gathered}
\mathcal{K}(\lambda)=-2 \sin [2 \lambda], \\
\Delta(\lambda)=-2 \sin \left[\frac{5}{6}+2 \lambda\right],
\end{gathered}
$$

therefore the eigenvalues are $\lambda_{k}=(6 k \pi-5) / 12, k \in \mathbb{Z}$.

Let $E$ and $E_{G}$ denote the absolute errors associated with the results of classical sinc method and sinc-Gaussian method, respectively. In Table 1, we give comparison between the absolute error of sinc-Gaussian and the classical sinc-method.

In Table 2, we observe that the approximation $\lambda_{k, N}$ and the exact solution $\lambda_{k}$ are all inside the interval $\left[a_{-}, a_{+}\right]$for different values of $\varepsilon$.

Example 4.2. In this example we consider the system

$$
\begin{gathered}
u_{2}^{\prime}(x)-p(x) u_{1}(x)=\lambda u_{1}(x), \\
u_{1}^{\prime}(x)+p(x) u_{2}(x)=-\lambda u_{2}(x), \quad x \in[-1,0) \cup(0,1],
\end{gathered}
$$


Table 2: The approximation $\lambda_{k, N}$ and the exact solution $\lambda_{k}$ are all inside the interval $\left[a_{-}, a_{+}\right]$for different values of $\varepsilon$.

\begin{tabular}{lcccc}
\hline$\lambda_{k}$ & Exact $\lambda_{k}$ & {$\left[a_{-}, a_{+}\right], \varepsilon=10^{-2}$} & {$\left[a_{-}, a_{+}\right], \varepsilon=10^{-5}$} & $\lambda_{k, N}$ \\
\hline$\lambda_{-2}$ & -3.55825932025645 & {$[-3.59648,-3.52003]$} & {$[-3.55829,-3.55822]$} & -3.5582593202564 \\
$\lambda_{-1}$ & -1.98746299346156 & {$[-2.02569,-1.94923]$} & {$[-1.98750,-1.98746]$} & -1.98746299346155 \\
$\lambda_{0}$ & -0.41666666666666 & {$[-0.454895,-0.37843]$} & {$[-0.416704,-0.416628]$} & -0.41666666666666 \\
$\lambda_{1}$ & 1.15412966012822 & {$[1.115901,1.19235]$} & {$[1.15409,1.15416]$} & 1.15412966012823 \\
$\lambda_{2}$ & 2.72492598692312 & {$[2.68669,2.76315]$} & {$[2.72488,2.72496]$} & 2.72492598692315 \\
\hline$\|\mathcal{U}\|_{\infty}=67.9396, \beta_{N}(0)=1.2155$. & &
\end{tabular}

Table 3: The absolute errors of sinc-Gaussian and the classical sinc-method with $N=20, h=0.3, \omega=$ 1.2708 .

\begin{tabular}{lccccc}
\hline$\lambda_{k}$ & Sinc & Exact $\lambda_{k}$ & Sinc-Gaussian $\lambda_{k, N}$ & $E$ & $E_{G}$ \\
\hline$\lambda_{-2}$ & -3.29646018123751 & -3.29645993245731 & -3.29645993245735 & $2.48 \times 10^{-7}$ & $4.21 \times 10^{-14}$ \\
$\lambda_{-1}$ & -1.7256638185270 & -1.7256636056624 & -1.7256636056621 & $2.12 \times 10^{-7}$ & $2.68 \times 10^{-13}$ \\
$\lambda_{0}$ & -0.154867300702813 & -0.154867278867517 & -0.154867278867127 & $2.18 \times 10^{-8}$ & $3.89 \times 10^{-13}$ \\
$\lambda_{1}$ & 1.41592898901685 & 1.41592904792737 & 1.41592904792752 & $5.89 \times 10^{-8}$ & $1.44 \times 10^{-13}$ \\
$\lambda_{2}$ & 2.98672509121710 & 2.9867253747222 & 2.98672537472225 & $2.83 \times 10^{-7}$ & $1.86 \times 10^{-14}$ \\
\hline
\end{tabular}

subject to

$$
\begin{array}{cc}
\sqrt{3} u_{1}(-1)+u_{2}(-1)=0, & u_{1}(1)+\sqrt{3} u_{2}(1)=0, \\
u_{1}\left(0^{-}\right)-3 u_{1}\left(0^{+}\right)=0, & u_{2}\left(0^{-}\right)-3 u_{2}\left(0^{+}\right)=0
\end{array}
$$

where

$$
p_{1}(x)=p_{2}(x)=p(x)= \begin{cases}x, & x \in[-1,0) \\ x^{2}+1, & x \in(0,1]\end{cases}
$$

$\alpha=\pi / 3, \beta=\pi / 6$ and $\delta=3$.

Direct calculations give

$$
\begin{gathered}
\mathcal{K}(\lambda)=3 \sin \left[\frac{\pi}{6}-2 \lambda\right], \\
\Delta(\lambda)=\frac{3}{2}\left(\cos \left[\frac{5}{6}+2 \lambda\right]-\sqrt{3} \sin \left[\frac{5}{6}+2 \lambda\right]\right),
\end{gathered}
$$

therefore the eigenvalues are $\lambda_{k}=((6 k+1) \pi-5) / 12, k \in \mathbb{Z}$.

Table 3 gives comparison between the absolute error of sinc-Gaussian and the classical sinc-method. Moreover, in Table 4 the approximation $\lambda_{k, N}$ and the exact solution $\lambda_{k}$ are all inside the interval $\left[a_{-}, a_{+}\right]$for different values of $\varepsilon$. 
Table 4: The approximation $\lambda_{k, N}$ and the exact solution $\lambda_{k}$ are all inside the interval $\left[a_{-}, a_{+}\right]$for different values of $\varepsilon$.

\begin{tabular}{lcccc}
\hline$\lambda_{k}$ & Exact $\lambda_{k}$ & {$\left[a_{-}, a_{+}\right], \varepsilon=10^{-2}$} & {$\left[a_{-}, a_{+}\right], \varepsilon=10^{-5}$} & $\lambda_{k, N}$ \\
\hline$\lambda_{-2}$ & -3.29645993245731 & {$[-3.322822,-3.270097]$} & {$[-3.296486,-3.296433]$} & -3.29645993245735 \\
$\lambda_{-1}$ & -1.7256636056624 & {$[-1.752025,-1.699301]$} & {$[-1.725689,-1.725637]$} & -1.7256636056621 \\
$\lambda_{0}$ & -0.154867278867517 & {$[-0.181229,-0.128505]$} & {$[-0.154893,-0.1548409]$} & -0.154867278867127 \\
$\lambda_{1}$ & 1.41592904792737 & {$[1.389566,1.442291]$} & {$[1.415902,1.415955]$} & 1.41592904792752 \\
$\lambda_{2}$ & 2.9867253747222 & {$[2.960363,3.013087]$} & {$[2.986699,3.013087]$} & 2.98672537472225 \\
\hline$\|\mathcal{U}\|_{\infty}=531.544, \beta_{N}(0)=1.22382$. & & &
\end{tabular}

\section{Acknowledgment}

This paper was funded by the Deanship of Scientific Research (DSR), King Abdulaziz University, Jeddah. The authors, therefore, acknowledge with thanks DSR technical and financial support.

\section{References}

[1] R. P. Agarwal, Boundary Value Problems for Higher Order Differential Equations, World Scientific, Singapore, Singapore, 1986.

[2] A. H. Bhrawy and A. S. Alofi, "A Jacobi-Gauss collocation method for solving nonlinear Lane-Emden type equations," Communications in Nonlinear Science and Numerical Simulation, vol. 17, no. 1, pp. 62-70, 2012.

[3] A. H. Bhrawy, A. S. Alofi, and S. I. El-Soubhy, "Spectral shifted Jacobi tau and collocation methods for solving fifth-order boundary value problems," Abstract and Applied Analysis, Article ID 823273, 14 pages, 2011.

[4] A. H. Bhrawy and W. M. Abd-Elhameed, "New algorithm for the numerical solutions of nonlinear third-order differential equations using Jacobi-Gauss collocation method," Mathematical Problems in Engineering, Article ID 837218, 14 pages, 2011.

[5] E. H. Doha and W. M. Abd-Elhameed, "Efficient solutions of multidimensional sixth-order boundary value problems using symmetric generalized Jacobi-Galerkin method," Abstract and Applied Analysis, vol. 2012, Article ID Article ID 749370, 19 pages, 2012.

[6] E. H. Doha, A. H. Bhrawy, and R. M. Hafez, "A Jacobi dual-Petrov-Galerkin method for solving some odd-order ordinary differential equations," Abstract and Applied Analysis, Article ID 947230, 21 pages, 2011.

[7] A. Imani, A. Aminataei, and A. Imani, "Collocation method via Jacobi polynomials for solving nonlinear ordinary differential equations," International Journal of Mathematics and Mathematical Sciences, Article ID 673085, 11 pages, 2011.

[8] O. N. Litvinenko and V. I. Soshnikov, The Theory of Heterogeneous Lines and their Applications in Radio Engineering, Radio, Moscow, Russia, 1964.

[9] M. H. Annaby and R. M. Asharabi, "Computing eigenvalues of boundary-value problems using sincGaussian method," Sampling Theory in Signal and Image Processing, vol. 7, no. 3, pp. 293-311, 2008.

[10] M. H. Annaby and R. M. Asharabi, "Approximating eigenvalues of discontinuous problems by sampling theorems," Journal of Numerical Mathematics, vol. 16, no. 3, pp. 163-183, 2008.

[11] R. Kh. Amirov, "On a system of Dirac differential equations with discontinuity conditions inside an interval," Ukrainian Mathematical Journal, vol. 57, no. 5, pp. 601-613, 2005.

[12] V. Kotelnikov, On the Carrying Capacity of the Either and Wire in Telecommunications, Material for the First All-Union Conference on Questions of Communications, Izd. Red. Upr. Svyazi RKKA, Moscow, Russia, 1933.

[13] C. E. Shannon, “Communication in the presence of noise," vol. 37, pp. 10-21, 1949.

[14] E. T. Whittaker, "On the functions which are represented by the expansion of the interpolation theory," Proceedings of the Royal Society of Edinburgh A, vol. 35, pp. 181-194, 1915. 
[15] P. L. Butzer, G. Schmeisser, and R. L. Stens, "An introduction to sampling analysis," in Non Uniform Sampling: Theory and Practices, F. Marvasti, Ed., pp. 17-121, Kluwer, New York, NY, USA, 2001.

[16] M. A. Kowalski, K. A. Sikorski, and F. Stenger, Selected Topics in Approximation and Computation, SIAM, Philadelphia, Pa, USA; Oxford University Press, New York, NY, USA, 1995.

[17] J. Lund and K. L. Bowers, Sinc Methods for Quadrature and Differential Equations, Society for Industrial and Applied Mathematics (SIAM), Philadelphia, Pa, USA, 1992.

[18] F. Stenger, "Numerical methods based on Whittaker cardinal, or sinc functions," SIAM Review, vol. 23, no. 2, pp. 165-224, 1981.

[19] F. Stenger, Numerical Methods Based On Sinc and Analytic Functions, Springer-Verlag, New York, NY, USA, 1993.

[20] M. H. Annaby and M. M. Tharwat, "On computing eigenvalues of second-order linear pencils," IMA Journal of Numerical Analysis, vol. 27, no. 2, pp. 366-380, 2007.

[21] M. H. Annaby and M. M. Tharwat, "Sinc-based computations of eigenvalues of Dirac systems," Behaviour \& Information Technology, vol. 47, no. 4, pp. 699-713, 2007.

[22] M. H. Annaby and M. M. Tharwat, "On the computation of the eigenvalues of Dirac systems," Calcolo, In press.

[23] A. Boumenir, "Higher approximation of eigenvalues by the sampling method," Behaviour E Information Technology, vol. 40, no. 2, pp. 215-225, 2000.

[24] A. Boumenir, "Sampling and eigenvalues of non-self-adjoint Sturm-Liouville problems," SIAM Journal on Scientific Computing, vol. 23, no. 1, pp. 219-229, 2001.

[25] P. L. Butzer and R. L. Stens, "A modification of the Whittaker-Kotelnikov-Shannon sampling series," Aequationes Mathematicae, vol. 28, no. 3, pp. 305-311, 1985.

[26] R. Gervais, Q. I. Rahman, and G. Schmeisser, "A bandlimited function simulating a duration-limited one," in Anniversary Volume on Approximation Theory and Functional Analysis, pp. 355-362, Birkhäuser, Basel, Switzerland, 1984.

[27] R. L. Stens, "Sampling by generalized kernels," in Sampling Theory in Fourier and Signal Analysis: Advanced Topics, J. R. Higgins and R. L. Stens, Eds., pp. 130-157, Oxford University Press, Oxford, UK, 1999.

[28] G. Schmeisser and F. Stenger, "Sinc approximation with a Gaussian multiplier," Sampling Theory in Signal and Image Processing, vol. 6, no. 2, pp. 199-221, 2007.

[29] L. Qian, "On the regularized Whittaker-Kotel'nikov-Shannon sampling formula," Proceedings of the American Mathematical Society, vol. 131, no. 4, pp. 1169-1176, 2003.

[30] L. Qian and D. B. Creamer, "A modification of the sampling series with a Gaussian multiplier," Sampling Theory in Signal and Image Processing, vol. 5, no. 1, pp. 1-19, 2006.

[31] L. Qian and D. B. Creamer, "Localized sampling in the presence of noise," Applied Mathematics Letters, vol. 19, no. 4, pp. 351-355, 2006.

[32] M. M. Tharwat, A. H. Bhrawy, and A. Yildirim, "Numerical computation of the eigenvalues of a discontinuous Dirac system using the sinc method with error analysis," International Journal of Computer Mathematics, Submitted.

[33] B. M. Levitan and I. S. Sargsjan, "Introduction to spectral theory: self adjoint ordinary differential operators," in Translation of Mthematical Monographs, vol. 39, American Mathematical Society, Providence, RI, USA, 1975.

[34] B. M. Levitan and I. S. Sargsjan, Sturm-Liouville and Dirac Operators, Kluwer Academic, Dordrecht, The Netherlands, 1991.

[35] M. M. Tharwat, "Discontinuous Sturm-Liouville problems and associated sampling theories," Abstract and Applied Analysis, Article ID 610232, 30 pages, 2011. 


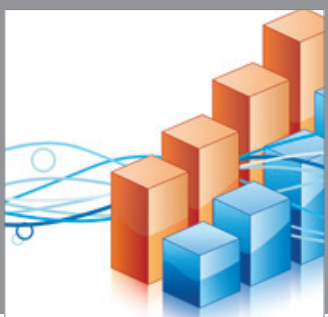

Advances in

Operations Research

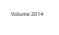

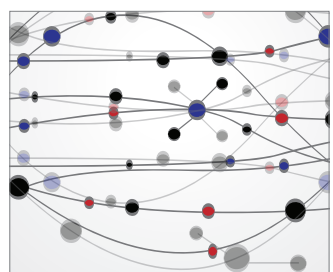

\section{The Scientific} World Journal
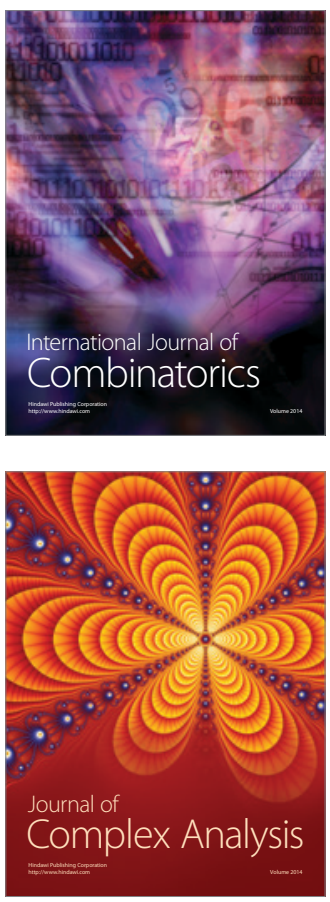

International Journal of

Mathematics and

Mathematical

Sciences
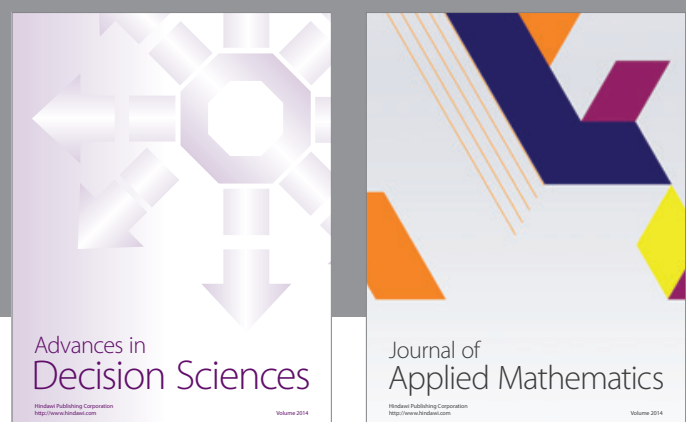

Journal of

Applied Mathematics
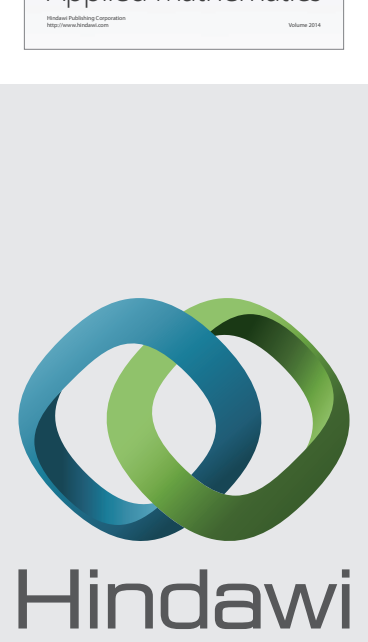

Submit your manuscripts at http://www.hindawi.com
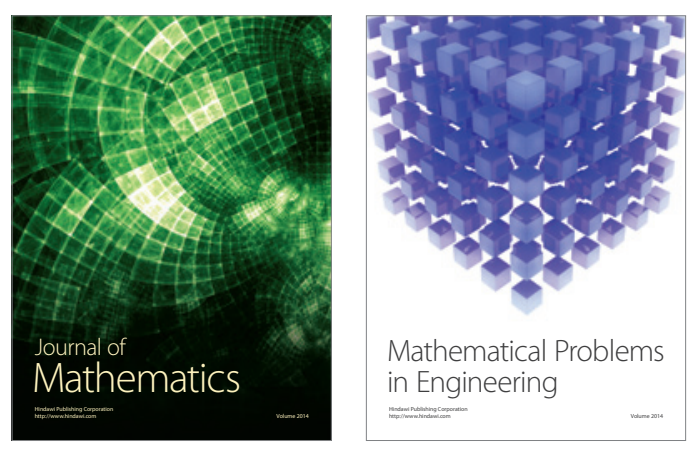

Mathematical Problems in Engineering
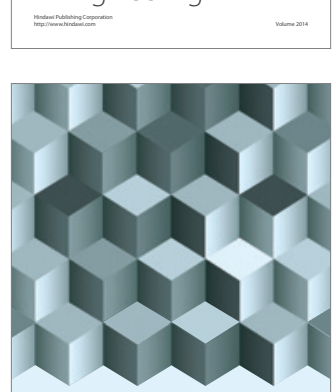

Journal of

Function Spaces
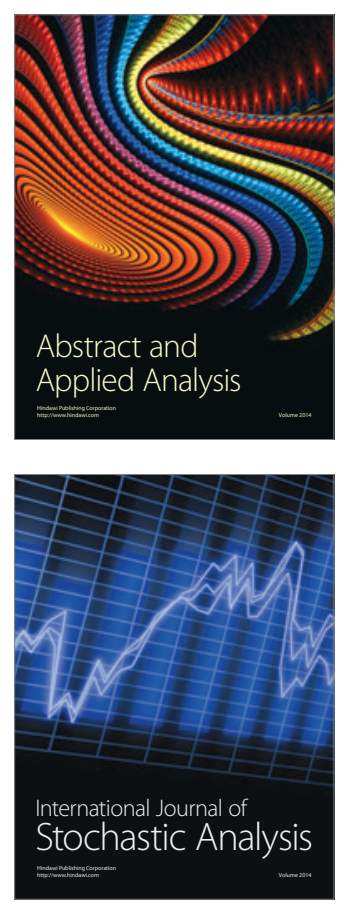

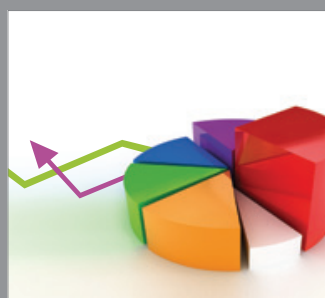

ournal of

Probability and Statistics

Promensencen
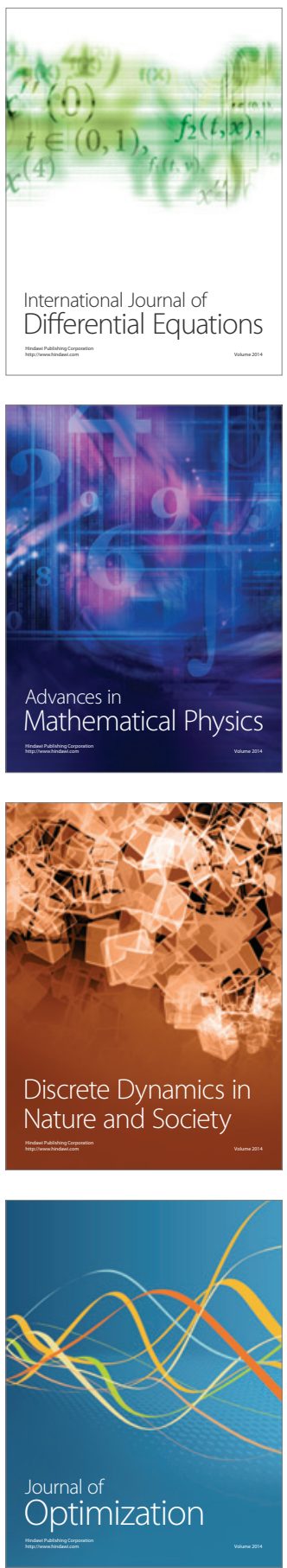